\title{
Criminologie
}

\section{La famille et la délinquance dans trois sphères culturelles}

\section{Emerson Douyon}

Volume 8, numéro 1-2, 1975

Délinquance juvénile au Québec

URI : https://id.erudit.org/iderudit/017039ar

DOI : https://doi.org/10.7202/017039ar

Aller au sommaire du numéro

Éditeur(s)

Les Presses de l'Université de Montréal

ISSN

0316-0041 (imprimé)

1492-1367 (numérique)

Découvrir la revue

Citer cet article

Douyon, E. (1975). La famille et la délinquance dans trois sphères culturelles. Criminologie, 8(1-2), 85-99. https://doi.org/10.7202/017039ar d'utilisation que vous pouvez consulter en ligne.

https://apropos.erudit.org/fr/usagers/politique-dutilisation/ 
LA FAMILLE ET LA DÉLINQUANCE DANS TROIS SPHERES CULTURELLES

Emerson Douyon 


\section{INTRODUCTION}

La famille est un système complet. Elle comprend une anatomie (cellules, sous-groupes, satellites, structure hiérarchique du pouvoir), une physologie (moyens de communications avec signaux, codes, symboles, langage verbal ou gestuel), une psychopathologie (mécanismes de défense, modes de neutralisation des conflits, symptômes et pathogénie). Il existe aussi une forme de thérapie appropriée au cas de la famille.

Selon Cattell (1950), on peut compter jusqu'à quatorze types de relations fondamentales dans la famille. Si nous y ajoutons les différentes modalités secondaires qui relient entre eux les membres, nous pouvons nous faire une idée approximative de la richesse des interactions au sein de la famille. Tout individu est le produit final de ce réseau relationnel diffus et complexe.

Lieu privilégié de rencontre entre le domaine du personnel et celui du socio-culturel, la famille initie à l'apprentissage et à la pratique de valeurs essentielles tels la tolérance à la frustration, l'identité personnelle, le sens de la propriété, de la solidarité, de la hiérarchie. D'un nourrisson polymorphe et asocial, elle en fait un " candidat à l'humanité ». Pour que la famille joue pleinement son rôle d'agent socialisateur et de matrice d'identification, il est impératif qu'elle forme un cadre de référence stable et une source de sécurité pour l'enfant.

Au mythe de la famille unie fonctionnant comme un bloc sans fissures, Jules Renard a opposé dans \& Poil de Carotte 》 une image sensiblement plus réaliste à certains égards pour un crimi- 
nologue. La famille serait plutôt cette * réunion forcée, sous le même toit, de quelques personnes qui ne peuvent pas se sentir $\$$. (cité par Porot 1963). D'un foyer irradiant l'amour elle peut se transformer en un nœud de vipères \$ ou en une structure aliénatrice d'invalidation. Dans ce cas la famille, loin d'engendrer un sentiment d'appartenance et une image consistante de soi, diffuse plutôt une impression obsédante d'aliénation. Vie de famille ou avenue royale vers la déviance, telle est la problématique.

\section{A. EVOLUTION OU CRISE DE LA FAMILLE OCCIDENTALE}

La famille, selon Philippe Ariès $(1962,1969)$ était autrefois une grande communauté intégrée, sans clivage marqué entre les adultes et les jeunes. Au cours de l'histoire, l'écart entre les parents et les enfants s'est considérablement élargi au point de créer ces «classes d'âges \ qui souffrent de plus en plus d'un sentiment d'aliénation au sein de la structure familiale.

D'autre part, l'emprise de la famille sur la quotidienneté de l'existence semble relativement récente. L'intervention de la famille jadis limitée aux périodes de crise interne devient davantage généralisée. Depuis la deuxième moitié du dix-huitième siècle, la famille se fait envahissante et annexe de nouveaux domaines dans la société capitaliste occidentale.

Où conduire cette évolution? Les plus pessimistes comme David Cooper (1972) pensent que c'est une structure appelée à disparaître et à être remplacée par d'autres «structures d'élevage >, comme les Kibboutz en Israël ou les nouvelles * communautés $\gg$ en Occident. On cherche de plus en plus des alternatives à la famille nucléaire patricentrique. On veut renoncer à \& fétichiser la consanguinité \& selon le mot de Cooper. Ce qu'on reproche essentiellement à la famille traditionnelle de l'occident, c'est d'être le prototype des institutions totalitaires. Au lieu que ce soit la Société qui agit sur la famille, c'est plutôt l'organisation familiale qui tend à se reproduire aux différents niveaux de la structure sociale, tels les écoles, l'université, le monde du travail.

On a coutume de dresser un parallèle entre l'aliénation mentale, l'aliénation familiale et l'aliénation sociale. Et on pense que c'est la famille qui est à la source de ces trois formes d'aliénation. On met en particulier l'accent sur l'aspect endoctrinement de la famille. Celle-ci est un * système colloïdal , qui étouffe à ce 
point l'autonomie, l'initiative, la créativité et la spontanéité, que dès la fin de l'enfance, l'individu y atteint un point de stagnation (Cooper 1972). Au lieu de se développer selon sa ligne à lui, celui-ci se trouve enfermé dans un certain nombre de rôles selon des scénarios fixés à l'avance.

Dans cette même perspective, Ronald Laing (1972) a institué une sévère critique de la politique de la famille. Celle-ci lui apparaît en effet comme une suite de drames qui tendent à reproduire des situations passées. Telle structure qui a prévalu dans la famille d'origine vise à se répéter dans la famille élective. Ainsi va le processus sur plusieurs générations. D'où un «échafaudage tubulaire répété à l'infini s (Hochmann, 1971). Sous prétexte que le fils tient du père qui ressemble au grand-père lequel était tout le portrait de l'arrière grand-père, on projette sur les jeunes de vieux rôles déjà assumés par des morts. Et progressivement chacun se trouve pris dans un noud dont il parvient difficilement à se dégager.

\section{B. LE RÔLE CRIMINOGÈNE DE LA FAMILLE}

La famille peut être perturbée dans sa structure ou dans son fonctionnement. Les vicissitudes de la vie familiale hypothèquent alors l'évolution affective de l'enfant. Entre la famille et le déviant, il existe une relation dialectique. Nous allons d'abord expliciter cette relation dans le cas de la délinquance et en référence au contexte Occidental et Québécois, (Garigue, 1970).

D'un point de vue structural, les recherches sur le rôle criminogène de la famille fournissent des résultats apparemment contradictoires. Le rapport classique des Glueck (1968) sur les familles des délinquants en Amérique du Nord indique dans quatrevingt-dix pour cent des cas étudiés la présence de valeurs déviantes au sein de ces familles. Les délinquants proviennent aussi deux fois plus souvent de familles dissociées que les non-délinquants.

La question de la dissociation familiale a été particulièrement soulignée dans la genèse de la délinquance. La rupture des liens familiaux causée par la mort, l'abandon, le divorce ou une forme quelconque de carence parentale, outre qu'elle provoque un effondrement de la structure d'autorité et des cadres de référence, peut également orienter vers la recherche de contact avec les agents criminogènes du milieu. La conduite délictuelle peut se présenter alors comme un exutoire aux tensions familiales. 
Toutefois đ'autres enquêtes comme celles de Christie, de Nye et Short (voir Muchielli, 1968), affirment respectivement soit que la majorité des délinquants viennent de familles normalement constituées, soit que ces familles gardent leur unité malgré leurs « dissensions » cachées. La désintégration familiale ouverte, publique, consacrée par le divorce par exemple, ne serait pas directement en cause dans le développement d'une personnalité dissociale.

À cet égard, il faut citer le témoignage autorisé de Louise Despert (1957) qui a dressé le tableau clinique des « enfants du divorce $\gg$. On y retrouve parmi les symptômes le sentiment de culpabilité, la réaction d'apathie, les difficultés scolaires, la régression émotionnelle, le refuge dans la maladie, les délits de compensation affective tels que fugue, prostitution, vol.

Le «divorce émotionnel qui a précédé la rupture légale serait le principal facteur de trouble. Car souvent un divorce légal peut jouer un rôle libérateur quant à une tension devenue insupportable pour les enfants. Le divorce n'exerce son effet dissolvant que lorsqu'il y a absence totale de compensation comme dans le cas d'un placement institutionnel. L'enfant peut alors se percevoir comme "un être de trop». Le divorce devient un alibi pour rationaliser les échecs et les troubles d'adaptation.

Il est possible cependant que les perturbations attribuées au divorce aient été la conséquence des réactions sociales d'autrefois. Puisque le divorce devient une institution normale au même titre que le mariage dans notre société, son acceptation par des secteurs de plus en plus étendus de la population en diminue "l'impact sur la personnalité des enfants. C'est à la lumière de toutes ces considérations qu'il faut réévaluer les apparentes contradictions des enquêtes statistiques et des rapports cliniques sur le rôle de la dissociation familiale dans la genèse de la délinquance.

Si l'on se place maintenant dans une perspective fonctionnelle pour expliquer les rapports entre la famille et la délinquance, on comprend mieux pourquoi la famille de type occidental est source d'aliénation et de déviance.

Ce qu'il faut d'abord retenir de cette structure, c'est que la famille constitue un milieu ambigu. La meilleure illustration de ce fait est celle du « dessin de la famille ». On sait que le dessin enfantin n'est pas une copie ou une reproduction pure et simple de la réalité, mais la projection d'un modèle intérieur. Or lors- 
qu'on demande aux frères et sœurs de représenter la famille, chacun projette son image intériorisée de la famille. Cette \& structure fantasmatique s peut être une fleur, une tombe, une prison ou un château, selon Laing (1972).

On voit donc qu'à côté de la famille objective, réelle, telle que perçue par les autres, il y a la famille subjective ou ce groupement intériorisé, tel que saisi et interprété par celui qui y a vécu. Dès lors ce n'est pas tant l'aspect objectif de la famille que certaines attitudes des parents telles que senties par l'enfant qu'il convient de retenir dans une explication causale de la délinquance.

Ces attitudes peuvent s'articuler autour de deux concepts de base, qui nous paraissent particulièrement éclairants pour comprendre les relations entre la famille et la délinquance.

Le premier de ces concepts est celui du \& postulat fusionnel \$ qui gouverne le fonctionnement familial pathologique. Ce postulat tel que défini par Jacques Hochmann (1971) énonce que « nous, membres de la famille, sommes tous identiques, pensons, sentons de même et sommes parfaitement transparents les uns aux autres $»$.

Le postulat fusionnel pose la famille comme une unité symbiotique où il est interdit à un membre de se différencier de l'ensemble. La famille est un bloc qui doit s'efforcer de masquer ses lézardes. En dépit des apparences contraires, elle doit fonctionner comme un mécanisme d'uniformisation et de "conformisation $\gg$.

Un exemple de ce postulat est la personnification des rôles. * La mère personnifie en l'un de ses enfants une partie d'ellemême, lui fait jouer un des rôles de son thêâtre intime s, dit Hochmann (1971, p. 168). Elle souhaite que chacun soit le prolongement des autres. Si tous les enfants étaient pareils et réalisaient les désirs ou idéaux des parents, ce serait tellement plus facile!

La délinquance figure une révolte contre cette tendance fusionnelle de la famille. Elle représente une tentative pour se différencier ou pour sortir de la confusion. Comme l'exprimait si bien un jeune de Boscoville à qui l'on venait d'apprendre qu'un autre membre de la famille commence à tourner mal. «Un délinquant dans la famille, c'est assez... Il veillera à ce qu'il n'y ait 
pas deux ». (Mailloux, 1972). Il veut à tout prix se différencier des autres et n'entend nullement devenir à son tour un pôle de fusion.

Le second concept auquel nous faisions allusion est celui de l'\& identité négative * ou du * mouton noir de la famille s. Selon Noël Mailloux (1972) au Québec, comme ailleurs en occident, les parents se font un portrait anticipé de l'enfant et de son identité future.

Cette attente peut être négative, c'est-à-dire, caractérisée par un manque de confiance. L'enfant est sans cesse confronté à une image dévalorisante de lui-même qu'il est amené progressivement à intérioriser. Il devient peu à peu le méchant, l'incapable, le mouton noir de la famille.

Une telle image projetée par les parents et de plus en plus assumée par l'enfant est cause d'angoisse. Pour se défendre contre cette angoisse existentielle, le jeune aura tendance à répéter de façon compulsive le comportement négatif attendu de lui.

L'identité négative sera diffusée plus tard aux autres milieux. A l'école l'enfant en retirera des bénéfices secondaires grâce à un début de réputation comme dur. Il se retire de la compétition, refusera de changer et aura tendance à interpréter tous ses échecs en fonction d'une constitution perverse précoce. Il deviendra le mauvais garçon exactement tel qu'on le lui avait souvent répété. * Çà fait cinq ans, me disait un adolescent, que ma mère me parlait du Mont-Saint-Antoine. Eh! bien, aujourd'hui j'y suis... je n'aurais jamais imaginé qu'elle pourrait deviner si juste.

On peut suggérer à un enfant soit un idéal du moi négatif (Mon Dieu, ça commence mal! J'espère que tu ne seras pas un vagabond), soit une conduite déviante (fais tes bagages et tâche de trouver mieux ailleurs). Beaucoup de vols et de fugues font suite à de pareilles interprétations ou invitations. Il arrive aussi qu'à l'occasion de la moindre faute, l'enfant se voit menacé d'une mauvaise fin comme son oncle, ou son cousin un tel qui jadis a fait la honte de la famille. Comment peut-on espérer qu'un enfant fasse quelque chose de bon après qu'on lui a répété : tu n'es bon à rien. Par leurs pronostics optimistes ou pessimistes, les parents conditionnent notre avenir. Comme l'a justement souligné un psychanalyste, nous devenons bons ou mauvais selon les fantasmes de nos parents à notre égard. 
Ronald Laing (1972) a systématisé ces différents schémas explicatifs de la délinquance de la manière suivante. On peut amener quelqu'un à incarner ses projections. Il suffit, comme dans l'hypnose, d'indiquer à l'enfant ce qu'il est et il prend place dans l'espace ainsi défini. On l'induit à être méchant en définissant comme méchant ce qu'il fait. *Les parents sont surpris par un enfant qui fait $x$ quand il lui disent de faire $y$ tout en lui suggérant qu'il est $x>$ (Laing, 1972, p. 101). Cette induction hypnotique conditionne l'apprentissage de la délinquance au sein de la famille.

\section{FAMILle, MODĖles CULTURELS ET DEVIANCE}

Dans le développement de toute société, la famille a toujours été considérée comme une modalité significative. Cependant il n'y a qu'en Occident où l'on entend parler d'une crise de la famille. Celle-ci serait responsable de toutes les formes d'aliénation et de révolte qui sont diffusées ensuite dans la société globale. Lorsque Cooper (1972) prétend que nous n'avons besoin ni de mère, ni de père, mais d'attention maternelle ou paternelle, il évoque sans doute, par delà ce paradoxe apparent, la possibilité d'autres \& structures d'élevage > qui compenseraient les ratages des fonctions parentales. À côté de la famille nucléaire, urbanisée, techniquement sophistiquée, compétitive, hiérarchisée, fondée sur la puissance paternelle et la politique du mâle au détriment du «Droit de la mère », telle que la société québécoise nous en offre un échantillon occidental parfait, il existe d'autres archétypes de la famille comme dans certains pays socialistes et le Tiers-Monde. Nous nous limiterons aux modèles du Kibboutz Israélien et du lignage africain pour fins de comparaison.

\section{LA COMMUNAUTÉ KIBBOUTZ}

La communauté agricole Kibboutz est une expérience d'éducation de groupe en milieu naturel. D'origine allemande et d'inspiration socialiste, elle dérive d'un mouvement de révolte des jeunes contre l'autoritarisme de la famille bourgeoise occidentale.

Le Kibboutz postule le développement harmonieux de la collectivité avant l'épanouissement de la personne. La famille n'est plus l'intermédiaire indispensable entre l'enfant et la société. Elle est remplacée par un ensemble relationnel plus large où le 
" groupe de pairs $\gg$ joue dès les premières semaines de la vie le premier rôle formateur et socialisateur assumé ailleurs par le couple parental. La projection d'une génération sur l'autre se trouve neutralisée à cause de cette solution de continuité entre les adultes et les jeunes.

L'éducation communautaire du Kibboutz est fondée sur une nouvelle éthique de la société. Ni compétition, ni propriété privée, ni niveau d'aspiration excédant les possibilités de l'être ou les limites de l'avoir. Aucune orthodoxie religieuse, aucun exclusivisme parental, mais égalitalisme absolu et liberté d'initiative. La famille traditionnelle ou monocellulaire à l'occidentale est bouleversée dans ses fondements essentiels.

A la famille unie s'est substituée la communauté unifiée. En vertu d'une délégation du pouvoir parental, le Kibboutz prime le groupe qui passe avant les parents. Où sont les crises d'antan, nées de l'interaction entre les parents et l'enfant et qui selon Erickson (1954) conditionnent le développement normal de la personnalité ?

Ces crises existent mais s'expriment selon des modalités différentes. La mère garde un rôle indispensable pour l'allaitement naturel. Ici se limite son importance. Dès le quatrième jour de leur naissance, les enfants vivent non dans une maison familiale mais dans un foyer de groupe. Ce maternage est multiple. Plus de mère captatrice symbolisant le mythe du sacrifice sur l'autel de la famille.

A côté de la mère nourricière qui se retire progressivement pour devenir intermittente, prennent place les figures changeantes des « metapelets * ou celles qui «prennent soin > des bébés et des jeunes avec compétence. Les relations durables avec la même personne sont modifiées. La metapelet, la mère et le groupe des pairs partagent respectivement les fonctions de dispenser les soins éducatifs, de combler les besoins affectifs et de fournir la source de sécurité. La mère n'étant plus accaparée par les soins physiques à donner et le souci des frustrations à infliger devient plus disponible pour améliorer la qualité de sa présence.

Quant au père, il a perdu son piédestal traditionnel. Sa paternité devient une œuvre partagée. Il est symboliquement le père de chaque enfant lequel est le fils du Kibboutz. Tous bénéficient également de son aide, de sa protection et de sa présence réconfortante. Au lieu d'être le rival craint et respecté, le bourreau 
familial ou le tyran domestique qui fait trembler femme et enfants sous sa double puissance mâle et paternelle comme l'archétype du père occidental le suggère trop souvent, $i l$ est et demeure avant tout le premier et le plus expérimenté des camarades. Il ne s'impose à personne et ne domine personne.

Dans cette nouvelle conception de la parenté, le concept d'autorité se trouve dilué. Le père ne personnifie plus la Loi ou la Règle de manière exclusive et privilégiée. La Metapelet devient le «porteur de l'interdit» et la pression du groupe des pairs a plus de poids que la parole du père. Plus qu'aux parents, c'est au Kibboutz que le jeune s'identifie. Cette structure de la parenté devient le dépositaire des premières valeurs.

Les résultats de l'éducation communautaire au Kibboutz sont controversés. Comparée à la personnalité des jeunes québécois, celle de l'enfant du Kibboutz peut apparaître plus matérialiste, plus prosaïque, moins démonstrative. Elle manque de relief et d'ouverture sur le monde extérieur. Le sens de l'intimité et le goût de l'introspection lui font défaut. Comme l'enfant du Kibboutz n'a jamais été seul, il est porté vers le groupe et l'action collective. C'est une personnalité très socialisée, mais dépourvue de mystère et de profondeur. Elle est uniformisée au niveau de la moyenne et ne frappe ni par ses déficiences ni par ses hypertrophies.

Tous les observateurs de la vie au Kibboutz ont été frappés par ce passif dans le bilan de leur évaluation. Par contre, tout en reconnaissant ce caractère de société hermétique peu ouverte aux innovations qui caractérise le Kibboutz, certains spécialistes de l'analyse de la conduite humaine, tels Bruno Bettelheim (1971), sont revenus enthousiasmés par cette expérience communautaire. L'enfant du Kibboutz serait un enfant heureux et bien adapté à la société égalitaire qui encadre ses expériences.

Le Kibboutz protège d'abord l'individu contre les effets négatifs du mauvais maternage ou du paternalisme excessif ou déficient de la famille occidentale. À la carence parentale partielle, elle supplée par un maternage diversifié dans un cadre unique, au sein d'un groupe permanent et stimulant. Puisqu'il n'y a ni attachement exclusif au couple parental, ni rivalité et ambivalence en fonction du sexe des parents, l'œedipe et ses effets dévastateurs sont neutralisés. Du même coup la rivalité fraternelle et la lutte pour les faveurs et les privilèges, au sein de la 
famille occidentale, se trouvent éliminés. Le surmoi n'est plus individuel, mais collectif. Le sentiment de culpabilité, qui procède ailleurs d'un processus d'intériorisation des défenses parentales, ne renvoie ici qu'aux valeurs du groupe.

Comme ces valeurs sont en accord avec celles des parents, celles de l'École et celles du milieu, elles engendrent une cohérence interne du Moi. Aussi ne trouve-t-on à l'intérieur du Kibboutz aucun type marqué de déviance, soit sous la forme de maladie mentale, soit sous la forme d'une conduite délictuelle ou de désengagement. Pour tous, la vie prend sens et valeur. Comme chacun y retrouve sa vraie place, personne ne connaît de crise d'identité, d'expérience d'aliénation, ni de penchant vers une culture de retrait, une sous-culture ou une contre-culture.

Bref, le Kibboutz, contrairement aux prévisions les plus pessimistes, accélère le processus de développement dans certains de ses aspects essentiels. Il suscite l'accès à une autonomie plus précoce et installe la coopération là où étaient la contrainte, la convoitise, le conflit et la compétition. Si cette expérience de vie communautaire n'est pas créatrice d'originalité, elle demeure une matrice d'équilibre et de normalité.

\section{E. LE GROUPE PARENTAL AFRICAIN}

La communauté Kibboutz peut être comparée à divers points de vue à la famille africaine traditionnelle. Celle-ci, contrairement à la cellule familiale occidentale, ne se réduit pas au père, à la mère et aux enfants. L'unité de vie sociale n'est pas le groupement domestique restreint mais le «clan » qui se subdivise en 《 lignages ». La parenté peut être patrilinéaire ou agnatique, matrilinéaire ou utérine.

Dans ces structures familiales, l'autorité n'est pas nécessairement détenue par le père. Elle peut, selon le cas, ressortir à la fonction de l'oncle maternel ou du doyen d'âge dans la communauté. De plus, la parenté africaine est dite classificatoire. Selon Nicolas (1968), cette structure réfère au «principe » selon lequel tous les membres d'une génération, au sein d'un groupe déterminé, sont considérés comme des frères ou des sœurs \$. Un individu peut posséder également plusieurs pères ou plusieurs fils. Cette parenté qui est à la fois cadre de résidence, unité politique, économique et religieuse est souvent modifiée par la polygamie qui y introduit d'autres modalités relationnelles. 
Henri Collomb (1965) a souligné avec raison la puissance du sentiment d'appartenance au groupe chez l'enfant africain. Dès sa naissance celui-ci est inséré dans un réseau relationnel qui déborde largement le cadre de la famille, telle que nous la connaissons en Occident. D'abord en contact physique avec la mère de façon plus intime que l'enfant du Kibboutz, le jeune africain sera plus tard pris en charge par la fratrie et la \& classe d'âge » qui l'aideront à passer au travers de l'Oedipe avec infiniment moins de difficultés que l'enfant de l'Occident. De plus, l'agressivité du jeune africain sera très tôt canalisée à travers un ensemble de rites et de coutumes claniques qui auront pour fonction de sécuriser l'individu et de le soumettre au contrôle du groupe parental élargi.

Comme l'enfant du Kibboutz, le fils ou la fille du lignage n'auront jamais connu ni carence affective, ni solitude, ni rejet ou abandon, ni aliénation ni crise d'identité. Son développement sera axé sur la solidarité et la fidélité au groupe. Dans les deux cas, la délinquance est un faux problème. Tant que la communauté de base reste intégrée et n'est pas contaminée par les formes d'aliénation introduites sous le couvert de l'urbanisation et du développement accéléré, elle sera exempte de ces multiples formes d'inadaption juvénile qui expliquent les périodiques remises en question de nos «structures d'élevage *.

\section{CONCLUSION}

La presse mondiale (voir, La Presse, 9 octobre 1974) a récemment fait écho au cas David. Il s'agit de cet enfant américain, de trois ans, qui souffre d'une affection génétique rare. $\mathrm{Ne}$ pouvant fabriquer les anticorps nécessaires à la défense de son organisme, il a été placé dès sa naissance dans un globe de plastique stérilisé relié à des pièces aseptisées. Or, cet enfant de l'avis des spécialistes, s'est développé d'une façon heureuse et équilibrée, en dépit de l'isolement intégral, des expériences constantes de privation sensorielle et d'absence de contact physique avec sa mère. Son univers familial se réduit, depuis trois ans, à cette sphère de plastique.

Voilà remise en question toute notre vision de «l'épigénèse , ou du développement de la personnalité. Spitz (1968), Bowlby (1954) et Erickson (1954) ont bien montré l'influence de la stimulation sensorielle, affective et sociale sur les premières 
années de l'existence en vue d'un développement ultérieur sain et harmonieux. Même si le cas rapporté est unique dans les annales de la médecine et des sciences humaines, il invite à de sérieuses réflexions sur plusieurs points, en particulier sur le rôle de la famille dans l'évolution psychologique.

Ce rôle nous apparaît de plus en plus controversé à la suite du rapport précité et de notre mise en relation de la famille et de la délinquance dans trois climats sociaux différents. Tout cela prouve qu'il existe d'autres modèles biologiques et sociaux de développement que ceux postulés par la psychanalyse ou dérivés de l'observation de la famille occidentale. L'essentiel est de mettre toute orthodoxie épistémologique entre parenthèses et de garder ouverte notre perspective sur d'autres faits inexplorés, ou sur d'autres valeurs à découvrir.

La «commune » chinoise elle aussi pourrait être citée comme modèle d'organisation familiale et sociale. Elle non plus, au même titre que la communauté Kibboutz ou le village africain, ne secrète pas la délinquance. Notre structure familiale, celle du Québec, comme celle du monde occidental dans son ensemble, recèle au contraire un redoutable potentiel criminogène.

Faut-il souhaiter un réaménagement dans notre mode « groupal » d'existence? Notre structure familiale n'est pas parfaite, celle des autres non plus. Mais les effets de notre côté sont si désastreux dans certains cas, qu'on peut commencer à regarder ailleurs comment s'y prennent les autres. Surtout pas de placage mécanique d'une structure d'ensemble sur une autre mais adaptation réciproque et nécessaire réajustement, telle devrait être la règle. On envie peut-être notre productivité, notre rythme de vie, notre élan vers la liberté, l'«expansivité » de notre personnalité. Nous envions certainement leur sagesse, leur sérénité, leur contrôle, et surtout leur modèle d'équilibre et d'adaptation. 


\section{BIBLIOGRAPHIE}

ARIES, P. (1962) : Des siècles d'enfance, Knopf.

ARIES, P. (1969) : - Wills, Tombs and families 》, New Society, no 356, p. 473-475, septembre 1969 .

BETTELHEIM, B. (1971) : les Enfants du rêve, une expérience d'éducation communautaire dans un Kibboutz d'Israël, Laffont.

CATTELL, R.B. (1950) : Personality, McGraw-Hill.

COLOMB, H. (1965) : Assistance psychiatrique en Afrique, Psychopathologie africaine, vol. I, $\mathrm{n}^{\circ} 1$.

COOPER, D. (1972) : Mort de la famille, Seuil.

DESPERT, Louise (1957) : les Enfants du divorce, P.U.F.

ERICKSON, E. (1954) : Enfance et sociêté, Delachaux.

GARIGUE, P. (1970) : la Vie familiale des Canadiens français, Presses de l'Université de Montréal.

GLUECK (1968) : Unravelling Juvenile Delinquency, Harvard Press.

HOCHMANN, J. (1971) : Pour une psychiatrie communautaire, Seuil.

LAING, R.D. (1972) : la Politique de la famille, Stock.

MAILLOUX, N. (1972) : Jeunes sans dialogue, Fleurus.

MUCHIELLI, R. (1965) : Comment ils deviennent délinquants, E.S.F.

NICOLAS, G. (1968) : La Société africaine et ses réactions à l'impact occidental, , in : l'Afrique contemporaine, Armand Colin.

POROT, M. (1963) : l'Enfant et les relations familiales, P.U.F.

SPITZ, R. (1968) : De la naissance à la parole. La première année de vie. P.U.F. 